OPEN ACCESS

Edited by:

Sabata Pierno,

Università Degli Studi di Bari Aldo

Moro, Italy

Reviewed by:

Anusha Ande,

University of Florida, United States

Sonia Maria Oliani,

Universidade Estadual Paulista Júlio

Mesquita Filho, Brazil

*Correspondence:

Mingxiang Yu

yu.mingxiang@zs-hospital.sh.cn

Xinhua Qu

Xinhua_qu@126.com

Specialty section

This article was submitted to

Translational Pharmacology,

a section of the journal

Frontiers in Pharmacology

Received: 01 May 2017

Accepted: 07 July 2017

Published: 25 July 2017

Citation:

Yang Y, Zhao C, Liang J, Yu M and QuX (2017) Effect of Dipeptidyl

Peptidase-4 Inhibitors on Bone

Metabolism and the Possible

Underlying Mechanisms.

Front. Pharmacol. 8:487.

doi: 10.3389/fphar.2017.00487

\section{Effect of Dipeptidyl Peptidase-4 Inhibitors on Bone Metabolism and the Possible Underlying Mechanisms}

\author{
Yinqiu Yang ${ }^{1}$, Chenhe $Z$ hao $^{1}$, Jing Liang ${ }^{1}$, Mingxiang $Y u^{1 *}$ and Xinhua $Q u^{2 *}$ \\ ${ }^{1}$ Department of Endocrinology, Zhongshan Hospital, Fudan University, Shanghai, China, ${ }^{2}$ Department of Orthopedics, \\ Shanghai Key Laboratory of Orthopaedic Implants, Shanghai Ninth People's Hospital, Shanghai Jiaotong University School \\ of Medicine, Shanghai, China
}

Diabetes mellitus has been demonstrated to be closely associated with osteoporosis. Accordingly, hypoglycemic therapy is considered effective in treating metabolic bone disease. Recently, the effects of dipeptidyl peptidase-4 (DPP-4) inhibitors, a new type of antidiabetic drug, on bone metabolism have been widely studied. This review mainly describes the effects of DPP-4 inhibitors on bone metabolism, including their effects on bone mineral density, bone quality, and fracture risk. In addition, the potential underlying mechanisms are discussed. Based on the current progress in this research field, DPP-4 inhibitors have been proved to reduce fracture risk. In addition, sitagliptin, a strong and highly selective DPP-4 inhibitor, showed its beneficial effects on bone metabolism by improving bone mineral density, bone quality, and bone markers. With regard to the potential underlying mechanisms, DPP-4 inhibitors may promote bone formation and reduce bone resorption through DPP-4 substrates and DPP-4-related energy metabolism. Vitamin D and other related signaling pathways also play a role in affecting bone metabolism. Although these assumptions are controversial, they provide a translational pharmacology approach for the clinical use of DPP-4 inhibitors in the treatment of metabolic diseases. Prior to the use of these drugs in clinic, further studies should be conducted to determine the appropriate type of DPP-4 inhibitor, the people who would benefit the most from this therapy, appropriate dose and duration, and the effects of the treatment.

Keywords: DPP-4 inhibitors, fracture risk, bone metabolism, bone formation, bone resorption

\section{INTRODUCTION}

It is well known that diabetes is associated with an increased incidence of osteoporosis, possibly mediated by the effects of insulin deficiency and hyperglycemia on the bone (Achemlal et al., 2005). Many researchers have shown that antidiabetic drugs may affect bone metabolism (Montagnani and Gonnelli, 2013; Jing and Zheng-ping, 2015). Recently, dipeptidyl peptidase-4 inhibitors (DPP4Is), a new type of antidiabetic drug widely used in clinical therapy, have received attention for their potential use in the treatment of osteoporosis.

DPP-4, also known as adenosine deaminase complexing protein-2 or cluster of differentiation 26 (CD26), is a serine protease expressed on the surface of most cell types. It selectively cleaves alanine and proline from polypeptide substrates, resulting in the inactivation of these substrates, including glucagon-like peptide 1 (GLP-1) and gastric inhibitory polypeptide (GIP) (Green et al., 2006; Idris and Donnely, 2007). 
Thus, the mechanism of DPP-4Is involves prolonging the half-life of these incretins (GLP-1 and GIP) and consequently promoting insulin secretion from $\beta$-pancreatic cells to improve glucose tolerance (Jing and Zheng-ping, 2015).

In the last decade, DPP-4Is such as vildagliptin, sitagliptin, linagliptin, and saxagliptin have been developed and approved for the treatment of diabetes. In the following review, we summarize the related study progress to date and introduce the potential mechanisms mediating the effects of DPP-4Is on the bone, with the aim of providing a translational pharmacology approach for the clinical application of DPP-4Is in osteoporosis.

\section{EFFECT OF DPP-4Is ON FRACTURE RISK}

A meta-analysis of 28 randomized clinical trials concluded that treatment with DPP-4Is reduced the risk of fractures when compared with placebo or other treatments (Mantel-Haenszelodds ratio $[\mathrm{HR}] 0.60,95 \%$ confidence interval $[\mathrm{CI}] 0.37-0.99, P$ $=0.045$; Monami et al., 2011). A German retrospective analysis revealed that in patients with type 2 diabetes (T2D), the use of DPP-4Is in combination with metformin significantly decreased the risk of developing bone fractures compared to metformin monotherapy ( $\mathrm{HR}=0.67,95 \%$ CI $0.54-0.84$; Dombrowski et al., 2017). Similar results were reported by another nationwide study in South Korea (Choi et al., 2016), which showed the efficacy of DPP-4Is in reducing bone fracture risks, implying their therapeutic potential in osteoporosis.

However, another meta-analysis revealed that there was no difference in the risk of fracture between DPP-4I users and controls (risk ratio 0.95; 95\% CI 0.83-1.10; $P=0.50$ ) even in subgroup analyses of different types of DPP-4Is, different controls, and different follow-up durations (Fu et al., 2016). This finding was also proved by another meta-analysis reported by Mamza et al. (2016) and a retrospective population-based cohort study (Driessen et al., 2017).

These differences in results may be due to the following reasons: (1) small sample sizes of randomized clinical trials included in the meta-analyses, which may lead to very low fracture incidences in both experimental and control groups; (2) in most of the studies analyzed, the definition of fracture was not clear and various pathological fractures were included, including high-energy fractures; and (3) patients with type 2 diabetes in clinical trials might have used other drugs simultaneously, which may affect the results of the meta-analysis. These problems should be addressed in future studies to provide a translational pharmacology approach for the clinical application of DPP-4Is in osteoporosis.

\section{EFFECT OF DPP-4IS ON BONE MINERAL DENSITY (BMD) AND BONE QUALITY}

A cross-sectional study of 744 postmenopausal women in China revealed that participants in the highest quartile of DPP-4 activity

Abbreviations: DPP-4, dipeptidyl peptidase-4; MSCs, mesenchymal stem cells; ERK, extracellular signal-regulated kinase. had lower BMD in the lumbar spine and femoral neck $(P<0.05$; Zheng et al., 2015). Another study conducted in obese women also demonstrated that DPP-4 activity negatively correlated with BMD ( $R=-0.288, P=0.038)$ in the spine (Kim and Cho, 2016), suggesting that the usage of DPP-4Is might improve BMD. However, this finding is still controversial according to a recent study (Carbone et al., 2017).

In animal studies, sitagliptin treatment was reported to improve the vertebral volumetric BMD (Kyle et al., 2011). This effect was also found to be dose-dependent (Cusick et al., 2013). With regard to bone quality, researches conducted in diabetic rats showed that sitagliptin significantly improved cortical bone volume, trabecular architecture, and bone strength (Kyle et al., 2011; Glorie et al., 2014). The results of these studies suggest the possibility that DPP-4I, especially sitagliptin, may be effective in treating osteoporosis and reducing the risk of fractures by improving $\mathrm{BMD}$ and bone quality. However, different results were obtained for other kinds of DPP-4I. MK-0626 was reported to have neutral effects on bone quality (Achemlal et al., 2005), whereas saxagliptin showed negative effects (Sbaraglini et al., 2014). Since adequate data on these drugs are still lacking, further studies in both animals and humans are needed to validate these arguments.

\section{EFFECT OF DPP-4IS ON BONE TURNOVER MARKERS}

\section{Biochemical Markers of Bone Turnover}

An analysis of 124 healthy postmenopausal women revealed a positive correlation between serum DPP-4 activity and parathyroid hormone level. From the results, we speculate that DPP-4Is can decrease parathyroid hormone level, and thus inhibit the release of calcium from the bone to blood and reduce bone destruction (Kim and Cho, 2016). Another study showed that compared to other antidiabetic drugs, DPP-4Is could significantly increase the serum concentration of 25-hydroxy vitamin D3 (25(OH)-D), which has been reported to regulate the concentration of calcium and phosphate in the bloodstream, thereby promoting the growth and remodeling of the bone. The level of 25(OH)-D was also associated with the duration of DPP4 I treatment and strength of DPP-4 inhibitory activity (Barchetta et al., 2016).

\section{Bone Formation Markers}

A cross-sectional study showed that serum DPP-4 was positively associated with the bone formation markers, bone-specific alkaline phosphatase (ALP) and osteocalcin (Notsu et al., 2016). Moreover, sitagliptin was found to significantly reduce alkaline phosphatase (ALP) level (Kubota et al., 2012; Hegazy, 2015). A non-significant decline in serum osteocalcin was also reported (Hegazy, 2015). However, the underlying reason was not wellexplained in these studies.

\section{Bone Resorption Markers}

In a study of 76 non-obese postmenopausal women, serum DPP-4 activity was found to be correlated with serum carboxyterminal telopeptide (CTX) levels $(R=0.284, P=0.016$; 
Choi et al., 2016). Similar findings were observed in a study conducted in diabetic animals, wherein sitagliptin-treated group had significantly lower serum CTX-I levels (Cusick et al., 2013). Urinary deoxypyridinoline, another bone resorption marker, was also found to be significantly reduced in postmenopausal diabetic women who received sitagliptin $(100 \mathrm{mg} /$ day $)$ for 12 weeks (Hegazy, 2015).

However, clinical trials using vildagliptin showed no changes in both bone formation and resorption markers (Bunck et al., 2012). Saxagliptin was found to increase osteoclastic tartrateresistant acid phosphatase activity (Sbaraglini et al., 2014).

In summary, among the different types of DPP-4Is, only sitagliptin was proved to affect bone turnover markers. This could be because sitagliptin has high inhibitory activity and selectivity for DPP-4 compared to other DPP-4Is. Therefore, to confirm the therapeutic potential of DPP-4Is in metabolic bone diseases, future studies should focus on sitagliptin and other strong DPP4Is. In addition, extending the follow-up time might help in studying the ameliorating effects of DPP-4Is. The published human and animal studies on the impact of DPP-4I on bone metabolism are described in Table 1.

\section{POTENTIAL MECHANISMS OF DPP-4IS ON BONE METABOLISM}

DPP-4Ts, in particular sitagliptin, showed their therapeutic potential in metabolic bone diseases by reducing fracture risk and improving BMD as well as bone turnover markers. The potential mechanisms underlying these effects are complicated. There are several possible ways through which DPP-4Is may affect bone metabolism, and these are summarized in Figure 1.

\section{DPP-4Is Affect Bone Metabolism by Lowering Glucose Level}

In diabetic patients, insulin deficiency and hyperglycemia seem to play a role in reducing bone formation. Advanced glycation end product (AGE) accumulation or AGE/RAGE (receptors for AGE) axis imbalance directly influences osteoblast activity by decreasing their number and function (Rico et al., 1989; Montagnani and Gonnelli, 2013). Moreover, hyperglycemia negatively affects osteocalcin production and the Wnt signaling pathways through an imbalance in osteoblast/osteoclast activity, leading to bone quality reduction as a global effect (Achemlal et al., 2005; Suzuki et al., 2005).

DPP-4Is can promote insulin secretion from $\beta$-pancreatic cells to improve glucose tolerance by prolonging the half-life of GLP1 and GIP (Jing and Zheng-ping, 2015), and they markedly decrease the negative effects of hyperglycemia on the bone.

\section{DPP-4ls Affect Bone Metabolism through DPP-4 Substrates GIP}

DPP-4 can degrade and inactivate polypeptide substrates, such as GIP, by selectively cleaving off alanine and proline (Idris and Donnely, 2007; Jing and Zheng-ping, 2015). Therefore, DPP-4 inhibition can prolong and enhance the effects of GIP on the bone.
In osteoblasts, GIP receptor (GIPR) activation after GIP binding can increase intracellular $\mathrm{Ca}^{2+}$ and cyclic adenosine monophosphate (cAMP) concentrations, along with ALP activity and type I collagen mRNA expression (Bollag et al., 2000; Baggio and Drucker, 2007). Moreover, the addition of GIP to cultured osteoblast precursors promotes their differentiation, increases their proliferation, and exhibits an anti-apoptotic activity in multipotent mesenchymal cells in the bone marrow (McIntosh et al., 1996).

GIPR, which is expressed in osteoclasts, is involved in downregulating bone resorption. In mature osteoclasts, GIP inhibits active osteoclast resorptive activity, as assessed by osteoclast pit formation assay, and decreases the expression of osteoclast-differentiation markers, such as the enzymes TRAP and cathepsin K and the M-CSF-receptor (Zhong et al., 2007).

Thus, DPP-4Is may promote bone formation and reduce bone loss by preventing GIP degradation.

\section{GLP-1}

Similar to GIP, DPP-4Is were reported to inhibit the degradation of GLP-1 by selectively cleaving alanine and proline (Idris and Donnely, 2007; Jing and Zheng-ping, 2015).

In mouse osteoblastic MC3T3-E1 cells, GLP-1 binding to its receptor increased osteocalcin and osteoprotegerin expression, decreased Runt-related transcription factor 2 (Runx2) expression, promoted osteoblast proliferation, and inhibited apoptosis (Nuche-Berenguer et al., 2010). These findings were also proved by an animal study, in which GLP-1 was found to markedly increase osteoblast number, as well as Runx2, ALP, and type I collagen levels in old rats with osteoporosis (Lamari et al., 1996). GLP-1 receptor is also expressed on the surface of osteocytes (Pereira et al., 2015), where it reduced the expression of sclerostin, a protein known to inhibit osteoblastic activity and stimulate catabolic actions on the bone (Kim et al., 2013).

With regard to bone resorption, GLP-1 receptors can be found anchored to the surface of primary osteoclasts. An animal study demonstrated a significant decrease in osteoclast number, CTX1 , and the urinary deoxypyridinoline/creatinine ratio after 16 weeks of GLP-1 use (Henriksen et al., 2003; Glorie et al., 2014). GLP-1 receptors can also be found on the surface of thyroid $\mathrm{C}$ cells, which promote the secretion of calcitonin through a cAMP-mediated pathway. Calcitonin inhibits osteoclast activity and prevents the release of calcium from the bone to the blood (Henriksen et al., 2009; Kim and Cho, 2016).

The positive effects of GLP-1 on BMD, bone strength, and bone architecture have been confirmed by animal studies (Achemlal et al., 2005; Mabilleau et al., 2013; Mansur et al., 2015). The various effects of GLP-1 on bone metabolism have been revealed in current studies, pointing out the anabolic effect of DPP-4 inhibition as well as the protective effects against bone loss.

\section{Others}

Other kinds of DPP-4 substrates also show their individual effects on bone metabolism, which can all be enhanced by DPP-4 inhibition. Stromal cell-derived factor- 1 alpha has been found to increase osteoblast proliferation and type-1 
TABLE 1 | Published human and animal researches on the impact of DPP-4ls on bone metabolism.

References Subjects Methods Main results

\begin{tabular}{ll}
\hline HUMAN & \\
$\begin{array}{l}\text { Monami et al., } \\
2011\end{array}$ & $\begin{array}{l}\text { A meta-analysis includes 28 RCTs in } \\
\text { T2D patients }\end{array}$ \\
$\begin{array}{l}\text { Dombrowski et al., } \\
\text { 1,262 T2D patients with an initial } \\
\text { prescription of metformin }\end{array}$ & 4,160 DPP-4l ever users vs. never users $(1: 1)$
\end{tabular}

Choi et al., $2016 \quad 207,558$ Subjects with antidiabetes prescriptions

Fu et al., $2016 \quad$ A meta-analysis includes 62 RCTs of 62,206 T2D patients

Mamza et al., 2016

Driessen et al., 2017

Zheng et al., 2015

Kim and Cho,

2016

Carbone et al., 2017

Barchetta et al., 2016

Notsu et al., 2016

Hegazy, 2015 40 Postmenopausal diabetic women

Kubota et al., 2012

Bunck et al., 201259 Drug-naïve patients with type 2 diabetes 204 Japanese men with T2DM

A meta-analysis includes 51 eligible RCTs of 36,402 participants

328,254 T2D patients with at least one prescription for a non-insulin antidiabetic drug

744 Postmenopausal women with normal glucose tolerance

124 Obese postmenopausal women

1,536 Male and female participants

295 Consecutive individuals with type 2 diabetes

The association between serum DPP4 and bone markers (BMD)

14 RCTs: DPP-4 inhibitor vs. an active comparator. ( $n=12,428)$

DPP-4 inhibitor users vs. other antidiabetic drug users

High DPP4 activity vs. low DPP4 activity

The association between BMD and DPP-4 activity

The association between BMD and DPP-4 activity

DPP4-Is(53\%) vs. control$$
\text { markers (BMD) }
$$

Fracture risk: a reduced fracture risk in DPP-4I users (MH-OR 0.60, 95\% Cl 0.37-0.99, $P=0.045$ )

Fracture risk: DPP4I use decreases the risk of developing bone fractures $(\mathrm{HR}=0.67,95 \% \mathrm{Cl}$ 0.54-0.84)

Fracture risk: the use of DPP-4ls could be associated with decreased risk of fracture

Fracture risk: no different risk of fracture (RR, 0.95; 95\% Cl, 0.83-1.10; $P=0.50$ ), even in subgroups using different type of DPP-4 inhibitor, different type of control, and different length of follow-up

Fracture risk: no significant association of fracture events with the use of DPP-4 inhibitor when compared with placebo (OR; 0.82, 95\% Cl $0.57-1.16, P=0.9)$ or compared against an active comparator (OR; 1.59, 95\% Cl 0.91-2.80, $P=0.9$ )

Fracture risk: the use of DPP-4 inhibitors was not associated with risk of any fracture (adjusted hazard ratio [HR] 0.99 [95\% confidence interval \{Cl\} 0.93-1.06])

BMD: Participants in the highest quartile of DPP4 activity had lower BMD (lumbar spine and femoral neck) compared with participants in the lowest quartile $(P<0.05)$

BMD: Serum DPP-4 activity was negatively correlated $\left(R=\hat{a}^{\wedge \prime} 0.288, P=0.038\right)$

BMD: In multivariable adjusted models, there was no association of plasma DPP-4 activity with BMD overall ( $p$ â\%॰ 0.55 for all) or in gender stratified analyses ( $p$ â\%o $¥ 0.23$ )

25(OH)D level: DPP4-Is-treated participants had significantly higher serum 25(OH)D levels than those undertaking other antidiabetic therapies (18.4 \pm 10.7 vs. $14.9 \pm 8.6 \mathrm{ng} / \mathrm{ml}, p=0.004)$, correlated with the duration and strength of DPP4 inhibitory

- ALP: positively associated with serum DPP-4

- Osteocalcin: positively associated with serum DPP-4

- Tartrate-resistant acid phosphatase 5b (TRACP5b): positively associated with serum DPP-4

- BMD: not changed

- Metformin (500 mg, bid) vs. sitagliptin (100 mg/d) - duration: 12 weeks

- ALP: the mean serum total ALP was significantly decreased in sitagliptin-treated group

- Osteocalcin: serum osteocalcin levels were nonsignificantly decreased gradually by $10 \%$ at 12 weeks in sitagliptin-treated group

- UDPD: urinary DPD decreased significantly and was then maintained at $28 \%$ decrease at 12 weeks in sitagliptin-treated group

ALP: decreased significantly after sitagliptin administration, from $255.3 \pm 93.0 \mathrm{IU} / \mathrm{L}$ at baseline to $240.3 \pm 86.1 \mathrm{IU} / \mathrm{L}$ at 4 weeks $(P<0.01)$ and $231.8 \pm 78.2 \mathrm{IU} / \mathrm{L}$ at 12 weeks $(P<0.01)$

CTX, not changed; ALP, not changed; calcium, not changed 
TABLE 1 | Continued

References Subjects Methods Main results

\section{ANIMALS}

Kyle et al., 2011

Male and female C57BL/6 mice

HFD-fed

Cusick et al., 2013 Non-parous female Sprague-Dawley rats

Sitagliptin (100, 300, or 500 mg/kg/day for 12 weeks) vs. blank control group

Pioglitazone vs. sitagliptin vs. genetic DPP-4 inactivation

- BMD: Sitagliptin treatment significantly improved vertebral volumetric BMD in female mice (not OVX).

- Bone quality: Sitagliptin significantly improved trabecular architecture and reduced trabecular separation in female mice (not OVX). OVX Dpp4/ mice exhibited significantly reduced femoral size and mechanics

BMD: BMD generally did not differ significantly between OVX-sitagliptin-treated animals and OVX-vehicle controls. However, there was significantly less BMD loss in lumbar vertebrae with increasing sitagliptin dose

Glorie et al., 201464 Male Wistar rats (2 diabetic and Sitagliptin vs. control two control groups)

MK-0626(4 g/kg) vs. control

Saxagliptin(2 mg/kg/day for 3 weeks) vs. contro

- Bone quality: sitagliptin attenuated trabecular bone loss and prevented cortical bone growth stagnation, resulting in stronger femora

- CTX: the serum levels of the resorption marker CTX-I were significantly lower in sitagliptin-treated group

Gallagher et al., Male wild type and diabetic 2014

Sbaraglini et al. 2014 muscle-lysine-arginine mice

Three-month-old male Sprague-Dawley rats
Bone quality: MK-0626 has neutral effects on cortical and trabecular bone

- Bone quality: a significant decrease in the femoral osteocytic and osteoblastic density of metaphyseal trabecular bone and a decrease in the average height of the proximal cartilage growth plate

- TRAP: an increase in osteoclastic tartrate-resistant acid phosphatase (TRAP) activity of the primary spongiosa

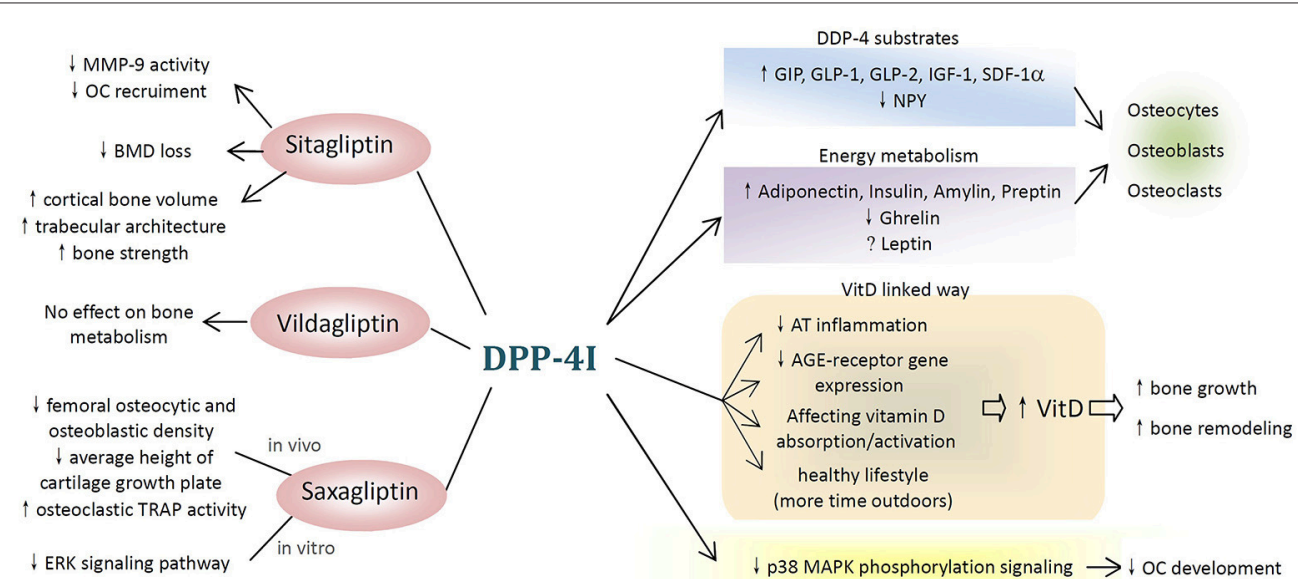

FIGURE 1 | Potential mechanisms of dipeptidyl peptidase-4 inhibitor (DPP-4I) on bone metabolism. (1) Different types of DPP-4ls affect bone metabolism differently. Sitagliptin have beneficial effects on bone resorption, bone mineral density, and bone quality, while vildagliptin shows no effect and saxagliptin negatively affects bone metabolism both in vivo and in vitro. (2) DPP-4ls attenuate the negative effects of hyperglycemia on the bone. (3) DPP-4ls affect bone cells and bone markers through DPP-4 substrates and DPP-4-related energy metabolism. (4) DPP-4 exerts inhibitory effects on bone metabolism through vitamin D-linked pathway. (5) Other possible pathways (MMP-9, matrix metallopeptidase-9; OC, osteoclast; BMD, bone mineral density; TRAP, tartrate-resistant acid phosphatase; ERK, extracellular signal-regulated kinase; GIP, gastric inhibitory polypeptide; GLP, glucagon-like peptide; SDF-1 $\alpha$, stromal cell-derived factor-1 alpha; NPY, neuropeptide Y; VitD, vitamin D; AT, adipose tissue; AGE, advanced glycation end products; p38 MAPK, p38 mitogen-activated protein kinase). 
collagen expression (Lisignoli et al., 2006). Increased earlystage osteoclast differentiation and reduced osteoclast apoptosis were also observed (Wright et al., 2005). GLP-2 was proved to attenuate bone resorption postprandially in various clinical studies (Haderslev et al., 2002; Henriksen et al., 2003, 2009).

\section{DPP-4ls Affect Bone Metabolism by Affecting Energy Metabolism}

A complex interaction exists between nutrition and bone metabolism, which involves many peptide molecules and adipokines, and this interaction is influenced by DPP-4.

\section{Leptin}

Leptin is predominantly secreted by adipocytes, and its secretion has been found to be associated with DPP-4 (Lamers et al., 2011). Leptin receptors are expressed in osteoblasts, and binding of leptin to the receptor promotes their proliferation and differentiation (Cornish et al., 2002). It has also been reported to reduce the production of receptor activator of nuclear factor- $\kappa$ B ligand (RANKL; Cornish et al., 2002) and increase osteoprotegerin expression, thereby reducing osteoclastogenesis (Holloway et al., 2002).

\section{Adiponectin}

Adiponectin is another peptide specifically secreted by adipocytes. It has been reported to promote the differentiation of osteoblasts as well as stimulate their activity ( $\mathrm{Wu}$ et al., 2014). In addition, adiponectin suppresses osteoclastic bone resorption (Oshima et al., 2005) and inhibits the differentiation of pre-osteoclasts (Ouchi et al., 2000). DPP-4 inhibition can attenuate the decrease in adiponectin receptor expression in patients with diabetes, thereby promoting bone formation and reducing bone resorption (Sakr, 2013).

\section{Ghrelin}

DPP-4 inhibition has been found to suppress active ghrelin (McIntosh et al., 1996), an appetite-stimulating hormone produced by the stomach, which stimulates the proliferation and differentiation of osteoblasts (Fukushima et al., 2005) as well as increases bone resorption and bone turnover in a fasted state (Oshima et al., 2005).

\section{DPP-4ls Affect Bone Metabolism through Vitamin D-Linked Pathway}

DPP-4Is significantly increased serum 25( $\mathrm{OH})$-D concentrations (Barchetta et al., 2016), which in turn led to the growth and remodeling of the bone. The possible mechanisms through which DPP-4Is increase vitamin D level are listed below.

\section{DPP-4 Modulates Adipose Tissue (AT) Inflammation}

AT represents the main site of vitamin $\mathrm{D}$ accumulation in humans and is a main target of vitamin D action. DPP-4 is highly expressed in adipocytes and resident macrophages/dendritic cells in inflamed AT (Röhrborn et al., 2015). In diabetic mice, sitagliptin administration has been reported to reduce AT inflammation (Röhrborn et al., 2015). It can be suggested that chronic DPP-4 inhibition modulates AT inflammation and stimulates the activation/mobilization of vitamin $\mathrm{D}$ from adipocytes into the bloodstream.

\section{DPP-4Is Inhibit RAGE Expression}

The photoconversion of provitamin $\mathrm{D}$ into vitamin $\mathrm{D}$ might be obstructed by cutaneous AGE accumulation. Notably, direct inhibition of DPP-4 activity by linagliptin was shown to prevent RAGE expression in keratinocytes (Schürmann et al., 2012; KrulPoel et al., 2015; Yamagishi et al., 2015), subsequently improving the local production of vitamin D.

\section{DPP-4ls Accelerate Absorption and Activation of Vitamin D}

Since DPP-4 is also expressed in the intestine, liver, and kidney (Röhrborn et al., 2015), a direct effect of DPP-4Is on vitamin D absorption and activation should be studied. DPP-4I treatment improves glycemic control, which can help lead a healthier lifestyle. Spending more time outdoors can also improve vitamin D levels.

In conclusion, DPP-4Is increase vitamin D level through different mechanisms. It has been well-demonstrated that vitamin $\mathrm{D}$ and its metabolites, especially calcitriol, can regulate the concentration of calcium and phosphate in the bloodstream, thereby promoting the growth and remodeling of the bone (Wolf, 2004).

\section{Other Pathways}

In addition to the above-mentioned mechanisms, there are other speculations about the mechanism of action of DPP4Is on bone metabolism that need to be validated. We have listed them below to offer several new possibilities for future studies.

\section{Metallopeptidase 9 (MMP-9)-Linked Pathway}

Baertsa et al. reported that sitagliptin could attenuate the increase in MMP-9 activity in patients with diabetes, probably because of its effect on inflammatory response. Therefore, the authors speculated that osteoclast recruitment by MMP-9 might be inhibited by sitagliptin (Baertsa et al., 2015). However, the definite underlying mechanisms remain unclear.

\section{P38 Mitogen-Activated Protein Kinase (MAPK) Phosphorylation Pathway}

Research suggests that the blockade of CD26 (DPP-4) signaling inhibits the p38 MAPK phosphorylation pathway, which is known to be an important step in early human osteoclast differentiation, and consequently impairs the development of human functional osteoclasts (Nishida et al., 2012, 2014). However, researchers examined the efficacy of thevidagliptin and found no significant inhibitory effect on human osteoclast differentiation or maturation. In addition, vidagliptin did not affect human osteoclast functions (Nishida et al., 2012). These contradictions in results need to be studied further.

\section{Extracellular Signal-Regulated Kinase (ERK) Signaling Pathway}

Most studies reported that DPP-4Is positively affect bone metabolism by promoting bone formation and reducing bone 
loss. However, saxagliptin is so far the only DPP-4I reported to have negative effects on bone metabolism, and its potential underlying mechanisms have been studied.

In vitro, saxagliptin was found to inhibit fetal bovine serum-, insulin-, and IGF1-induced ERK phosphorylation and proliferation in both mesenchymal stem cells (MSCs) and MC3T3-E1 pre-osteoblasts in the presence of growth factors. Furthermore, saxagliptin inhibited Runx2 and osteocalcin expression, type- 1 collagen production, and mineralization, and increased the expression of peroxisome proliferative activated receptor-gamma in the presence of fetal bovine serum (Sbaraglini et al., 2014).

The negative effects of orally administered saxagliptin on bone metabolism are probably due to the downregulation of the ERK signaling pathway for insulin as well as insulin-like growth factor 1 (IGF-1) in MSCs and the decreased osteogenic potential of these cells (Sbaraglini et al., 2014). However, this study presents certain limitations. The studies were conducted only in healthy rats and in vitro, and not in models of insulin resistance or type 2 diabetes. In addition, controlled clinical trials are necessary to verify the effects of saxagliptin on bone adequately.

\section{DISCUSSION}

This review could only partly answer how DPP-4Is affect bone fracture risk, BMD, and bone quality. The potential mechanisms underlying these effects were also partly revealed. Further studies are required before DPP-4Is can be considered for clinical use in metabolic bone disease. We have provided several suggestions about the future research direction as given below:

1) Focus on sitagliptin and other relatively strong DPP4 inhibitors. Although several kinds of DPP-4Is have been studied, we found that only sitagliptin, a relatively strong DPP-4 inhibitor, showed therapeutic potential in metabolic bone disease. The use of other drugs such as vildagliptin, saxagliptin, and MK-0626 for metabolic bone disease is controversial. Therefore, we suggest that future studies should focus on sitagliptin and other strong DPP-4 inhibitors.

2) More number of clinical trials should be performed in not only diabetic patients, but also other populations. From the study of potential mechanisms, we found that besides lowering glucose level, DPP-4Is might affect bone metabolism through other independent ways. Therefore, we suggest that more number of clinical trials should be performed to prove the effects of DPP-4Is in other populations.

3) The duration and follow-up time should be extended to find chronic changes. DPP-4Is might have chronic effects on bone metabolism, and to prove this, longer duration and follow-up time is required.

4) Dose-related studies to find effective dose with fewer side effects. The effects of DPP-4Is on bone metabolism might be dose dependent (Cusick et al., 2013). However, in most of the current studies, dosage was not regarded as a variable; therefore, we recommend more dose-related studies in the future. Side effects of DPP-4Is should also be taken in consideration.

5) Speculations about the molecular mechanisms of DPP-4 inhibitors in bone physiology should be analyzed further. Although we mentioned many potential mechanisms to explain the relationship between DPP-4Is and bone metabolism in our review, most of them are still speculations lacking adequate evidences.

\section{CONCLUSION}

Most researchers reported that DPP-4Is have beneficial effects on bone metabolism. Sitagliptin exerted its therapeutic potential in metabolic bone disease by reducing fracture risk and by improving BMD and bone turnover markers. However, studies with vildagliptin and saxagliptin have shown contradictory results.

DPP-4Is can affect bone metabolism through various mechanisms. First, DPP-4 inhibition may promote bone metabolism by lowering glucose level. Second, DPP-4 substrates and DPP-4-related energy metabolism may affect bone metabolism. Third, DPP-4Is might affect bone metabolism through vitamin D-linked and other related signaling pathways. However, the definite underlying mechanisms need to be elucidated further before we can truly provide a translational pharmacology approach for the clinical application of DPP-4Is for osteoporosis.

\section{AUTHOR CONTRIBUTIONS}

All authors listed have made a substantial, direct and intellectual contribution to the work, and approved it for publication.

\section{ACKNOWLEDGMENTS}

This work was supported by the National Natural Science Foundation for Youths (Grant No. 81401852), the Natural Science Foundation of Shanghai (No. 14ZR1424000), the "Chen Guang" Project of the Shanghai Municipal Education Commission and the Shanghai Education Development Foundation (No. 14CG14), and the Interdisciplinary Projects of Medicine and Engineering of Shanghai JiaoTong University (No. YG2015QN13).

\section{REFERENCES}

Achemlal, L., Tellal, S., Rkiouak, F., Nouijai, A., Bezza, A., Derouiche el, M., et al. (2005). Bone metabolism in male patients with type 2 diabetes. Clin. Rheumatol. 24, 493-496. doi: 10.1007/s10067-004-1070-9

Baertsa, L., Glorieb, L., Mahoc, W., Eelena, A., Verhulstb, A., D’Haeseb, P., et al. (2015). Potential impact of sitagliptin on collagen-derived dipeptides in diabetic osteoporosis. Pharmacol. Res. 100, 336-340. doi: 10.1016/j.phrs.2015.08.023

Baggio, L. L., and Drucker, D. J. (2007). Biology of incretins: GLP-1 and GIP. Gastroenterology 132, 2131-2157. doi: 10.1053/j.gastro.2007.03.054 
Barchetta, I., Cimini, F., A., Bloise, D., and Cavallo, M., G. (2016). Dipeptidyl peptidase-4 inhibitors and bone metabolism: is vitamin D the link? Acta Diabetol. 53, 839-844. doi: 10.1007/s00592-016-0882-9

Bollag, R. J., Zhong, Q., Phillips, P., Min, L., Zhong, L., Cameron, R., et al. (2000). Osteoblast-derived cells express functional glucosedependent insulinotropic peptide receptors. Endocrinology 141, 1228-1235. doi: 10.1210/endo.141. 3.7366

Bunck, M. C., Poelma, M., Eekhoff, E. M., Schweizer, A., Heine, R. J., Nijpels, G., et al. (2012). Effects of vildagliptin on postprandial markers of bone resorption and calcium homeostasis in recently diagnosed, well-controlled type 2 diabetes patients. J. Diabetes 4, 181-185. doi: 10.1111/j.1753-0407.2011.00 168.x

Carbone, L. D., Bủžková, P., Fink, H. A., Robbins, J. A., Bethel, M., Isales, C. M., et al. (2017). Association of DPP-4 activity with BMD, body composition, and incident hip fracture: the Cardiovascular health study. Osteoporosis Int. 28, 1631-1640. doi: 10.1007/s00198-017-3916-4

Choi, H. J., Park, C., Lee, Y.-K., Ha, Y.-C., Jang, S., and Shin, C. S. (2016). Risk of fractures and diabetes medications: a nationwide cohort study. Osteoporosis Int. 27, 2709-2715. doi: 10.1007/s00198-016-3595-6

Cornish, J., Callon, K. E., Bava, U., Lin, C., Naot, D., Hill, B. L., et al. (2002). Leptin directly regulates bone cell function in vitro and reduces bone fragility in vivo. J. Endocrinol. 175, 405-415. doi: 10.1677/joe.0.1750405

Cusick, T., Mu, J., Pennypacker, B. L., Li, Z., Scott, K. R., Shen, X., et al. (2013). Bone loss in the oestrogen-depleted rat is not exacerbated by sitagliptin, either alone or in combination with a thiazolidinedione. Diabetes Obes. Metab.15, 954-957. doi: $10.1111 /$ dom.12109

Dombrowski, S., Kostev, K., and Jacob, L. (2017). Use of dipeptidyl peptidase4 inhibitors and risk of bone fracture in patients with type 2 diabetes in Germany-a retrospective analysis of real-world data. Osteoporosis Int. 29, 1-8. doi: 10.1007/s00198-017-4051-y

Driessen, J. H., van den Bergh, J. P., van Onzenoort, H. A., Henry, R. M., Leufkens, H. G., and de Vries, F. (2017). Long-term use of dipeptidyl peptidase4 inhibitors and risk of fracture: a retrospective population-based cohort study. Diabetes Obes. Metab. 19, 421-428. doi: 10.1111/dom.12843

Fu, J., Zhu, J., Hao, Y., Guo, C., and Zhou, Z. (2016). Dipeptidyl peptidase-4 inhibitors and fracture risk: an updated meta-analysis of randomized clinical trials. Sci. Rep. 6:29104. doi: 10.1038/srep29104

Fukushima, N., Hanada, R., Teranishi, H., Fukue, Y., Tachibana, T., Ishikawa, H., et al. (2005). Ghrelin directly regulates bone formation. J. Bone Miner. Res. 20, 790-798. doi: 10.1359/JBMR.041237

Gallagher, E. J., Sun, H., Kornhauser, C., Tobin-Hess, A., Epstein, S., Yakar, S., et al. (2014). The effect of dipeptidyl peptidase-IV inhibition on bone in a mouse model of type 2 diabetes. Diabetes Metab. Res. Rev. 30, 191-200. doi: $10.1002 / \mathrm{dmrr} .2466$

Glorie, L., Behets, G., Lesley Baerts, J., De Meester, I., D’Haese, P., and Anja Verhulst, C. (2014). DPP IV inhibitor treatment attenuates bone loss and improves mechanical bone strength in male diabetic rats. Am. J. Physiol. Endocrinol. Metab. 307, 447-455. doi: 10.1152/ajpendo.00217.2014

Green, B. D., Flatt, P. R., and Bailey, C. J. (2006). Dipeptidyl peptidase IV (DPP IV) inhibitors: a newly emerging drug class for the treatment of type 2 diabetes. Diabetes Vasc. Dis. Res. 3, 159-165. doi: 10.3132/dvdr.2006.024

Haderslev, K. V., Jeppesen, P. B., Hartmann, B., Thulesen, J., Sorensen, H. A., Graff, J., et al. (2002). Short-term administration of glucagon-like peptide2. Effects on bone mineral density and markers of bone turnover in short-bowel patients with no colon. Scand. J. Gastroenterol. 37, 392-398. doi: $10.1080 / 003655202317316006$

Hegazy, S. K. (2015). Evaluation of the anti-osteoporotic effects of metformin and sitagliptin in postmenopausal diabetic women. J. Bone Miner. Metab. 33, 207-212. doi: 10.1007/s00774-014-0581-y

Henriksen, D. B., Alexandersen, P., Bjarnason, N. H., Vilsboll, T., Hartmann, B., Henriksen, E. E., et al. (2003). Role of gastrointestinal hormones in postprandial reduction of bone resorption. J. Bone Miner. Res. 18, 2180-2189. doi: 10.1359/jbmr.2003.18.12.2180

Henriksen, D. B., Alexandersen, P., Hartmann, B., Adrian, C. L., Byrjalsen, I., Bone, H. G., et al. (2009). Four-month treatment with GLP-2 significantly increases hip BMD: a randomized, placebo-controlled, dose-ranging study in postmenopausal women with low BMD. Bone 45, 833-842. doi: 10.1016/j.bone.2009.07.008
Holloway, W. R., Collier, F. M., Aitken, C. J., Myers, D. E., Hodge, J. M., Malakellis, M., et al. (2002). Leptin inhibits osteoclast generation. J. Bone Miner. Res. 17, 200-209. doi: 10.1359/jbmr.2002.17.2.200

Idris, I., and Donnely, R. (2007). Dipeptidyl peptidase-IV inhibitors: a major new class of oral antidiabetic drug. Diabetes Obes. Metabol. 9, 153-165. doi: 10.1111/j.1463-1326.2007.00705.x

Jing, D., and Zheng-ping, F. (2015). Effects of antidiabetic medicine on bone metabolism. Chin. J. Osteoporosis Bone Miner. Res. 3, 256-260. doi: 10.3969/j.issn.1674-2591.2015.03.013

Kim, J. Y., Lee, S. K., Jo, K. J., Song, D. Y., Lim, D. M., Park, K. Y., et al. (2013). Exendin- 4 increases bone mineral density in type 2 diabetic OLETF rats potentially through the down-regulation of SOST/sclerostin in osteocytes. Life Sci. 92, 533-540. doi: 10.1016/j.lfs.2013.01.001

Kim, S.-W., and Cho, E.-H. (2016). High levels of serum DPP-4 activity are associated with low bone mineral density in obese postmenopausal women. Endocrinol. Metab. 31, 93-99. doi: 10.3803/EnM.2016.31.1.93

Krul-Poel, Y. H., Agca, R., Lips, P., van Wijland, H., Stam, F., and Simsek, S. (2015). Vitamin D status is associated with skin autofluorescence in patients with type 2 diabetes mellitus: a preliminary report. Cardiovasc. Diabetol.14:89. doi: $10.1186 / \mathrm{s} 12933-015-0250-\mathrm{z}$

Kubota, A., Maeda, H., Kanamori, A., Matoba, K., Jin, Y., and Minagawa, F., et al. (2012). Pleiotropic effects of sitagliptin in the treatment of type 2 diabetes mellitus patients. J. Clin. Med. Res.4, 309-313. doi: 10.4021/jocmr1061w

Kyle, K. A., Willett, T. L., Baggio, L. L., Drucker, D. J., and Grynpas, M. D. (2011). Differential effects of PPAR-\{gamma\} activation versus chemical or genetic reduction of DPP-4 activity on bone quality in mice. Endocrinology 152 , 457-467. doi: 10.1210/en.2010-1098

Lamari, Y., Boissard, C., Moukhtar, M. S., Jullienne, A., Rosselin, G., Garel, J. M., et al. (1996). Expression of glucagon-like peptide 1 receptor in a murine C cell line: regulation of calcitonin gene by glucagon-like peptide 1. FEBS Lett. 393 , 248-252. doi: 10.1016/0014-5793(96)00895-2

Lamers, D., Famulla, S., Wronkowitz, N., Hartwig, S., Lehr, S., Ouwens, D. M., et al. (2011). Dipeptidyl peptidase 4 is a novel adipokine potentially linking obesity to the metabolic syndrome. Diabetes $60,1917-1925$. doi: $10.2337 / \mathrm{db}$ 10-1707

Lisignoli, G., Toneguzzi, S., Piacentini, A., Cristino, S., Grassi, F., Cavallo, C., et al. (2006). CXCL12 (SDF-1) and CXCL13 (BCA-1) chemokines significantly induce proliferation and collagen type I expression in osteoblasts from osteoarthritis patients. J. Cell. Physiol. 206, 78-85. doi: 10.1002/jcp. 20435

Mabilleau, G., Mieczkowska, A., Irwin, N., Flatt, P. R., and Chappard, D. (2013). Optimal bonemechanical and material properties require a functional glucagon-like peptide-1 receptor. J. Endocrinol. 219, 59-68. doi: 10.1530/JOE-13-0146

Mamza, J., Marlin, C., Wang, C., Chokkalingam, K., and Idris, I. (2016). DPP4 inhibitor therapy and bone fractures in people with Type 2 diabetes - a systematic review and meta-analysis. Diabetes Res. Clin. Pract. 116, 288-298. doi: 10.1016/j.diabres.2016.04.029

Mansur, S. A., Mieczkowska, A., Bouvard, B., Flatt, P. R., Chappard, D., and Irwin, N. G. (2015). Mabilleau, stable incretin mimetics counter rapid deterioration of bone quality in type 1diabetes mellitus. J. Cell. Physiol. 230, 3009-3018. doi: $10.1002 /$ jcp. 25033

McIntosh, C. H., Wheeler, M. B., Gelling, R. W., Brown, J. C., and Pederson, R. A. (1996). GIP receptors and signal-transduction mechanisms. Acta Physiol. Scand. 157, 361-365. doi: 10.1046/j.1365-201X.1996.44267000.x

Monami, M., Dicembrini, I., Antenore, A., and Mannucci, E. (2011). Dipeptidyl peptidase-4 inhibitors and bone fractures: a meta-analysis of randomized clinical trials. Diabetes Care 34, 2474-2476. doi: 10.2337/dc11-1099

Montagnani, A., and Gonnelli, S. (2013). Antidiabetic therapy effects on bone metabolism and fracture risk. Diabetes Obes. Metab. 15, 784-791. doi: $10.1111 /$ dom. 12077

Nishida, H., Madokoro, H., Suzuki, H., Sakamoto, M., Morimoto, C., and Yamada, T. (2012). Targeting CD26 with humanized monoclonal antibody, as a novel approach to inhibit human osteoclast differentiation and subsequent bone resorption. Blood 120:1348.

Nishida, H., Suzuki, H., Madokoro, H., Hayashi, M., Morimoto, C., Sakamoto, M., et al. (2014). Blockade of CD26 signaling inhibits human osteoclast development. J. Bone Miner. Res. 29, 2439-2455. doi: 10.1002/jbmr.2277 
Notsu, M., Kanazawa, I., Tanaka, S., Yamaguchi, T., and Sugimoto, T. (2016). Serum dipeptidyl peptidase- 4 is associated with multiple vertebral fractures in type 2 diabetes mellitus. Clin. Endocrinol. 84, 332-337. doi: 10.1111/cen.12971

Nuche-Berenguer, B., Portal-Nunez, S., Moreno, P., Gonzalez, N., Acitores, A., Lopez-Herradon, A., et al. (2010). Presence of a functional receptorfor GLP1 in osteoblastic cells, independent of the cAMP-linked GLP-1 receptor. J. Cell. Physiol. 225, 585-592. doi: 10.1002/jcp.22243

Oshima, K., Nampei, A., Matsuda, M., Iwaki, M., Fukuhara, A., Hashimoto, J., et al. (2005). Adiponectin increases bone mass by suppressing osteoclast and activating osteoblast. Biochem. Biophys. Res. Commun. 331, 520-526. doi: 10.1016/j.bbrc.2005.03.210

Ouchi, N., Kihara, S., Arita, Y., Okamoto, Y., Maeda, K., Kuriyama, H., et al. (2000). Adiponectin, an adipocyte-derived plasma protein, inhibits endothelial NF-kappaB signaling through a cAMP-dependent pathway. Circulation 102, 1296-301. doi: 10.1161/01.CIR.102.11.1296

Pereira, M., Jeyabalan, J., Jorgensen, C. S., Hopkinson, M., Al-Jazzar, A., Roux, J. P., et al. (2015). Chronic administration of Glucagon-like peptide-1 receptor agonists improves trabecular bone mass and architecture in ovariectomised mice. Bone 81, 459-467. doi: 10.1016/j.bone.2015.08.006

Rico, H., Hernandez, E. R., Cabranes, J. A., and Gomez-Castresana, F. (1989). Suggestion of a deficient osteoblastic function in diabetes mellitus: the possible cause of osteopenia in diabetics. Calcif. Tissue Int. 45, 71-73. doi: 10.1007/BF02561404

Röhrborn, D., Wronkowitz, N., and Eckel, J. (2015). DPP4 in diabetes. Front. Immunol. 6:386. doi: 10.3389/fimmu.2015.00386

Sakr, H. F. (2013). Effect of sitagliptin on the working memory and reference memory in type 2 diabetic Sprague-Dawley rats: possible role of adiponectin receptors 1. J. Physiol. Pharmacol. 64, 613-623.

Sbaraglini, M. L., Molinuevo, M. S., Sedlinsky, C., Schurman, L., and McCarthy, A. D. (2014). Saxagliptin affects long-bone microarchitecture and decreases the osteogenic potential of bone marrow stromal cells. Eur. J. Pharmacol. 727, 8-14. doi: 10.1016/j.ejphar.2014.01.028

Schürmann, C., Linke, A., Engelmann-Pilger, K., Steinmetz, C., Mark, M., Pfeilschifter, J., et al. (2012). The dipeptidyl peptidase-4 inhibitor linagliptin attenuates inflammation and accelerates epithelialization in wounds of diabetic ob/ob mice. J. Pharmacol. Exp. Ther. 342, 71-80. doi: 10.1124/jpet.111.191098

Suzuki, K., Kurose, T., Takizawa, M., Maruyama, M., Ushikawa, K., Kikuyama, M., et al. (2005). Osteoclastic function is accelerated in male patients with type 2 diabetes mellitus: the preventive role of osteoclastogenesis inhibitory factor/osteoprotegerin (OCIF/OPG) on the decrease of bone mineral density. Diabetes Res. Clin. Pract. 68, 117-125. doi: 10.1016/j.diabres.2004.08.006

Wolf, G. (2004). The discovery of vitamin D: the contribution of Adolf Windaus. J. Nutr. 134, 1299-1302.

Wright, L. M., Maloney, W., Yu, X., Kindle, L., Collin-Osdoby, P., and Osdoby, P. (2005). Stromal cellderived factor-1 binding to its chemokine receptor CXCR4 on precursor cells promotes the chemotactic recruitment, development and survival of human osteoclasts. Bone 36, 840-853. doi: 10.1016/j.bone.2005.01.021

Wu, Y., Tu, Q., Valverde, P., Zhang, J., Murray, D., Dong, L. Q., et al. (2014). Central adiponectin administration reveals new regulatory mechanisms of bone metabolism in mice. Am. J. Physiol. Endocrinol. Metab. 306, E1418-E1430. doi: 10.1152/ajpendo.00048.2014

Yamagishi, S., Fukami, K., and Matsui, T. (2015). Crosstalk between advanced glycation end products (AGEs)-receptor RAGE axis and dipeptidyl peptidase4 -incretin system in diabetic vascular complications. Cardiovasc. Diabetol. 14, 2. doi: 10.1186/s12933-015-0176-5

Zheng, T., Yang, L., Liu, Y., Liu, H., Yu, J., Zhang, X., et al. (2015). Plasma DPP4 activities are associated with osteoporosis in postmenopausal women with normal glucose tolerance. J. Clin. Endocrinol. Metab. 100, 3862-3870. doi: 10.1210/jc.2015-2233

Zhong, Q., Itokawa, T., Sridhar, S., Ding, K. H., Xie, D., and Kang, B., et al. (2007). Effects of glucose-dependent insulinotropic peptide on osteoclast function. Am. J. Physiol. Endocrinol. Metab. 292, E543-E548. doi: 10.1152/ajpendo.00364.2006

Conflict of Interest Statement: The authors declare that the research was conducted in the absence of any commercial or financial relationships that could be construed as a potential conflict of interest.

Copyright (c) 2017 Yang, Zhao, Liang, Yu and Qu. This is an open-access article distributed under the terms of the Creative Commons Attribution License (CC $B Y)$. The use, distribution or reproduction in other forums is permitted, provided the original author(s) or licensor are credited and that the original publication in this journal is cited, in accordance with accepted academic practice. No use, distribution or reproduction is permitted which does not comply with these terms. 